\title{
Influence of a multideficient diet from northeastern Brazil on resting blood pressure and baroreflex sensitivity in conscious, freely moving rats
}

F.M.F. Monteiro, S. Lahlou, J.A. Albuquerque and A.M.S. Cabral

\author{
Departamento de Fisiologia e Farmacologia, \\ Universidade Federal de Pernambuco, Recife, PE, Brasil
}

\section{Correspondence \\ S. Lahlou \\ Departamento de Fisiologia \\ e Farmacologia \\ Centro de Ciências Biológicas, UFPE \\ 50670-901 Recife, PE \\ Brasil \\ Fax: + 55-81-271-8976 \\ E-mail: lahlou@npd.ufpe.br \\ Presented in part at the XIII Annual Meeting of the Federação de \\ Sociedades de Biologia Experimental, Caxambu, M G, Brazil, August, 26-29, 1998.}

Research supported by FACEPE (No. APQ 0045-4.01-97) and Universidade Federal de Pernambuco.

Received May 5, 2000 Accepted December 12, 2000

\section{Abstract}

The "regional basic diet" or RBD is a multideficient diet (providing $8 \%$ protein) which is known to produce dietary deficiencies in some populations in northeastern Brazil. The present study investigated the effects of RBD-induced malnutrition on resting blood pressure and baroreflex sensitivity in conscious rats. Malnourished rats were obtained by feeding dams the RBD during mating and pregnancy (RBD1 group) or during nursing and a 10-day period after weaning (RBD2 group). At 90 days of age, only RBD-2 rats weighed significantly $(\mathrm{P}<0.001)$ less than control rats born to dams fed a standard commercial diet (23\% protein) during pregnancy and nursing. Baseline mean arterial pressure and heart rate of both RBD-1 and RBD-2 rats were comparable to those of controls. The slopes for both reflex bradycardia and tachycardia $(\mathrm{bpm} / \mathrm{mmHg})$ induced by intravenous phenylephrine and sodium nitroprusside, respectively, were unchanged in either RBD-1 (-2.08 \pm 0.11 and $-3.10 \pm 0.43$, respectively) or RBD-2 ( -2.32 \pm 0.30 and $-3.73 \pm 0.53$, respectively) rats, when compared to controls $(-2.09 \pm 0.10$ and $-3.17 \pm 0.33$, respectively). This study shows that, after a prolonged period of nutritional recovery, the patterns of resting blood pressure and baroreflex sensitivity of both pre- and postnatally malnourished rats were similar to those of controls. The decreased body weight and the tendency to increased reflex tachycardia in RBD2 rats may suggest that this type of maternal malnutrition during lactation is more critical than during pregnancy.

\section{Introduction}

Epidemiological studies have implicated maternal protein-calorie deficiency as an important public health problem in underdeveloped countries (1-3), including Brazil (4). Maternal malnutrition is known to be the most significant factor for high infant and postnatal mortality rates in these countries (5). Experi-
Key words

- Multideficient diet

- Intrauterine and postnatal malnutrition

- Blood pressure

- Baroreflex sensitivity

- Conscious rats mental studies have shown that pre- or postnatal nutritional manipulations may program adult size, metabolism, blood lipids, diabetes, obesity, blood pressure, glomerular hypertrophy, atherosclerosis, behavior, and learning ( 6 , for references see 7). The clinical consequences of malnutrition depend on several factors, including the severity and duration of nutritional deficiencies. 
Epidemiological and experimental studies designed to examine the relationship between intrauterine environment (as reflected by birth weight) and variation in adult blood pressure have yielded contradictory results. For instance, several human studies have shown that birth weight is inversely associated with blood pressure in childhood, as well as in adult life (8-14), but this association has not been confirmed by all investigators (15-17). In their experimental studies, Langley and co-workers (18) showed that rats exposed to a low-protein diet in utero exhibited lower body weight at birth and higher systolic blood pressure at 4 weeks of age. This hypertension was reported to persist into adulthood (19). Using a similar feeding protocol, Tonkiss et al. (20) were unable to corroborate the latter experimental findings. Furthermore, no differences were detected in basal blood pressure of adult rats that had received a low-protein diet during perinatal life (21). It has been also shown that chronic caloric restriction in adulthood had no effect on blood pressure in normotensive rats (22), but decreased this parameter in spontaneously hypertensive rats (23). However, the baroreflex responsiveness of both calorie-restricted groups was enhanced $(22,23)$. Given the above conflicting data and the paucity of information about the role of other nutrients in malnutrition-induced cardiovascular disease, the effects of feeding a multideficient diet during pregnancy or nursing on resting blood pressure, heart rate (HR), and baroreceptor control to HR were studied in conscious 3-month-old rats. The diet employed herein is deficient in protein, lipids and vitamins, as well as in sodium and other minerals (24) and was prepared according to the diet consumed by populations living in the area of sugar-cane cultivation in coastal Pernambuco, northeastern Brazil. Pioneer studies have indicated that this experimental diet, known as the "regional basic diet" or RBD, produces in rats a type of malnutrition similar to that prevalent among children from this region of Brazil, namely an association with nutritional dwarfism, with some clinical signs of marasmus (24). Other studies have demonstrated that RBD results in deficient glycemic regulation (25), reduction in sciatic nerve conduction velocity (26) and blunted propagation of spreading depression (27).

\section{Material and Methods}

\section{Animals}

Male and virgin female Wistar rats (initial weight 240-300 g) were kept under conditions of constant temperature $\left(23 \pm 2^{\circ} \mathrm{C}\right)$ on a standard light/dark cycle (12-h/12-h), with free access to food and water.

\section{Diets}

The ingredients $(\mathrm{g} / 100 \mathrm{~g})$ of the multideficient diet used herein were beans (Phaseolus vulgaris; 18.34), manioc flour (Manihot esculenta; 64.81), dried and salted meat (3.74), and sweet potato (Ipomaea batatas; 12.76). The diet was prepared in our laboratory as follows: all ingredients (except manioc flour) were cooked, dehydrated for 24$60 \mathrm{~h}$ (according to the type of ingredient) at $60^{\circ} \mathrm{C}$ and pulverized. Each component was mixed with manioc flour by humidifying. Meat fat $(0.35 \%)$ was then added, and the mixture was shaped into balls which were dehydrated for $24 \mathrm{~h}$ at $60^{\circ} \mathrm{C}$. The centesimal composition of the RBD, which was determined by the Department of Nutrition, Federal University of Pernambuco, is given in Table 1. The caloric adequacy of the RBD was calculated to be about $316 \mathrm{kcal}$ per $100 \mathrm{~g}$. Standard pellet chow (Purina do Brasil Ltd., São Paulo, SP, Brazil) was used as control diet.

\section{Feeding protocol}

During the mating period, all female and 
male rats were fed a standard balanced diet, except those belonging to group 1 (see below) which received the RBD. Throughout this period, the detection of sperm in the vagina was taken to indicate that fertilization had occurred. Fertilized females were then immediately transferred to individual cages and divided into three groups. Group 1 was fed the RBD during pregnancy. Group 2 was fed the RBD during nursing, and group 3, which served as the control, was fed the standard diet during pregnancy and nursing. All groups had free access to water and diets. Females in group 1 were placed on a standard diet within 2-12 h after giving birth and remained on this diet throughout suckling. Offspring of all mothers were weaned at 25 days of age. After weaning, offspring born to mothers in groups 1 and 3 were placed on the standard diet until 12 weeks of age. Ten days after weaning, offspring born to mothers in group 2 were switched to the standard diet and remained on it until 12 weeks of age. The body weight of offspring in all groups was determined weekly from the 1st to the 12th week of age. In order to minimize the putative risk of cannibalism, birth body weight was determined only in 6 offspring belonging to group 1 and compared to that of offspring belonging to the control group. In addition, daily food intake of some control and malnourished mothers was determined from pregnancy to weaning.

\section{Catheterization procedure}

The day before they reached 90 days of age, male rats born to mothers that had received the RBD during mating and pregnancy (RBD-1) or during nursing and the 10day period following weaning (RBD-2) and those born to mothers receiving the control diet during pregnancy and nursing (CNT) were anesthetized with ether, and two vascular catheters (PE-10 fused to PE-50) were implanted as follows: one catheter was inserted into the abdominal aorta via the left femoral artery for the recording of arterial blood pressure and the other was inserted into the inferior vena cava via the left femoral vein for drug administration. The catheters were filled with heparinized (125 IU/ $\mathrm{ml}$ ) isotonic saline and exteriorized at the dorsal neck level. Immediately after surgery, rats received an intramuscular injection of penicillin (24,000 IU). Postoperatively, the rats were housed individually in plastic cages and allowed to recover for $24 \mathrm{~h}$ before any circulatory experiments. All experiments were performed on conscious, freely moving rats.

\section{Recording of mean arterial pressure and heart rate}

At the time of the experiment, animals were maintained in a quiet room and the arterial catheter was connected to a blood pressure transducer (Statham P23 Db) coupled to a Hewlett Packard model 4574A polygraph (Hewlett Packard, Palo Alto, CA, USA). Mean arterial pressure (MAP) was calculated as diastolic $+[$ (systolic-diastolic) $/$ 3] pressure and HR was calculated from the blood pressure tracing.

Table 1 - Composition $(\mathrm{g} / \mathrm{g} \%$ ) of the multideficient synthetic and standard commercial diets.

Composition determined from the cooked multideficient diet.

\begin{tabular}{lcr}
\hline & $\begin{array}{c}\text { Standard } \\
\text { commercial diet }\end{array}$ & $\begin{array}{c}\text { Multideficient } \\
\text { diet }\end{array}$ \\
\hline Protein & 23.00 & 7.87 \\
Carbohydrates & 44.50 & 69.24 \\
Lipids & 2.50 & 0.80 \\
Fibers & 7.21 & 8.00 \\
Minerals & & \\
Ca ${ }^{+}$ & 1.80 & 0.95 \\
Mg $^{2+}$ & 0.32 & 0.44 \\
$\mathrm{~K}^{+}$ & 1.15 & 0.54 \\
$\mathrm{Na}^{+}$ & 0.36 & 0.25 \\
$\mathrm{Humidity}^{+}$ & 13.00 & 13.00 \\
$\mathrm{kcal} / 100 \mathrm{~g}$ & 292.50 & 315.64
\end{tabular}




\section{Experimental protocol}

In order to assess the effects of RBDinduced malnutrition on baseline cardiovascular parameters and baroreflex sensitivity, the following protocol was used. The venous catheter was connected to a long polyethylene tube which leads out of the top of the cage. This aimed to minimize animal manipulation and to allow the investigator to be out of the animal's sight. After a rest period of 30-45 min, basal MAP and HR values were determined and their maximum changes elicited by $i v$ injection of increasing bolus $(50 \mu \mathrm{l})$ doses of phenylephrine (PHE, 0.25-4 $\mu \mathrm{g} / \mathrm{kg}$ ) or sodium nitroprusside (SNP, 0.5-8 $\mu \mathrm{g} / \mathrm{kg}$ ) were assessed in each control and malnourished rat. PHE infusions were started first, and after MAP and HR returned to control baseline, SNP infusions were begun. In preliminary experiments, successive $i v$ injections of a similar volume of saline evoked nonsignificant effects on both baseline MAP and HR. Before each injection of PHE or SNP, MAP and HR were first allowed to return to their respective baseline values obtained at the beginning of the experiment. For each rat, the baroreflex responsiveness, as reflected by the ratio of the peak change in HR to the peak change in MAP (slope), was determined for both PHE and SNP using a linear regression analysis. The mean slope was calculated by averaging the baroreflex slope for each individual experiment. This investigation conformed to the "Guide for the Care and Use of Laboratory Animals" published by the US National Institutes of Health (NIH Publication No. 85-23, revised 1985).

\section{Determination of cardiac hypertrophy}

After the circulatory experiments, animals of all groups were killed by cervical dislocation and the hearts were excised. The hearts were then rinsed with saline and blotted with filter paper. They were weighed, dried for $48 \mathrm{~h}$ in a desiccator at $70^{\circ} \mathrm{C}$, and reweighed (28). The heart dry weight to body weight ratio was used as an index of cardiac hypertrophy, as previously reported (28-30).

\section{Statistical analysis}

All results are reported as mean \pm SEM. Changes in absolute MAP and HR with respect to individual baseline values were computed. One-way analysis of variance (ANOVA) and the unpaired Student $t$-test were employed to assess differences in baseline MAP, HR and body weight between the different groups. The statistical significance of MAP and HR responses to PHE or SNP was determined by the paired Student $t$-test. Comparisons between control and malnourished rats were performed using the unpaired Student $t$-test and Mann-Whitney U-test. Groups of data were compared by one-way (doses) or two-way (treatment $\mathrm{x}$ weeks or treatment $\mathrm{x}$ doses) ANOVA. Differences were considered to be statistically significant at $\mathrm{P}<0.05$.

\section{Drugs}

(-)-Phenylephrine hydrochloride and sodium nitroprusside were purchased from Sigma Chemical Co. (St. Louis, MO, USA) and were dissolved in saline. Solutions were prepared daily and SNP solutions were stored in the dark. Heparin (Laboratoires Léo S.A., Montigny-Le-Bretonneux, France) was used as the commercially available injectable solution. Penicillin $G$ benzathine salt (Lafepe, Recife, PE, Brazil) was dissolved in saline.

\section{Results}

\section{Effects of maternal RBD on body weight of offspring and heart weight}

Birth weight was significantly $(\mathrm{P}<0.001$, unpaired Student $t$-test) reduced in RBD-1 
rats $(4.55 \pm 0.23 \mathrm{~g}, \mathrm{~N}=6)$ when compared to CNT rats $(6.75 \pm 0.18 \mathrm{~g}, \mathrm{~N}=6)$. The time courses of body weight of RBD-1, RBD-2 and CNT rats from the 1 st to the 12 th week of age are shown in Figure 1. Throughout this monitoring period, no significant differences in body weight were observed between RBD- 1 and CNT rats ( $\mathrm{P}>0.05$, twoway ANOVA). However, two-way ANOVA revealed that this parameter was significantly $(\mathrm{P}<0.001)$ lower in $\mathrm{RBD}-2$ rats than in controls. This reduction in body weight was significant at all ages studied $(\mathrm{P}<0.01$, unpaired Student $t$-test). When RBD-2 rats were placed on a standard balanced diet by the 5 th week of age, they displayed a weekly rate of weight gain similar to that observed in CNT rats (Figure 1); however, the body weight of RBD-2 rats remained significantly reduced at the end of the 12-week monitoring period ( $\mathrm{P}<0.01$, unpaired Student $t$-test), and was approximately $75 \%$ of that of controls. Both wet and dry heart weights were significantly reduced in RBD- 1 and RBD-2 rats $(\mathrm{P}<0.05$, unpaired Student $t$-test), when compared to control rats (Figure $2 \mathrm{~A}$ and $\mathrm{B}$ ). However, the heart dry weight to body weight ratio of RBD-1 and RBD-2 rats was not statistically different $(\mathrm{P}>0.05$, unpaired Student $t$-test $)$ from that observed in CNT rats (Figure 2C).

\section{Effects of maternal RBD on baseline MAP and HR values}

Baseline MAP and HR recorded for RBD-1 (112 $\pm 4 \mathrm{mmHg}$ and $385 \pm 15 \mathrm{bpm}$, respectively, $\mathrm{N}=9)$ and RBD-2 (102 \pm 3 $\mathrm{mmHg}$ and $366 \pm 8 \mathrm{bpm}$, respectively, $\mathrm{N}=$ 10) rats were not significantly different ( $\mathrm{P}>0.05$, unpaired Student $t$-test) from those recorded for CNT rats $(110 \pm 4 \mathrm{mmHg}$ and $357 \pm 12$ bpm, respectively, $N=9$ ). In both malnourished groups, baseline diastolic and systolic arterial pressure values were also comparable to those recorded for well-nourished rats $(\mathrm{P}>0.05$, unpaired Student $t$-test) (data not shown).

\section{Effects of maternal RBD on baroreflex sensitivity}

In both RBD- 1 and RBD-2 rats, increasing bolus doses of $i v$ injected PHE and SNP evoked dose-dependent increases and decreases in MAP, respectively $(\mathrm{P}<0.05$, oneway ANOVA). These responses were significant with respect to baseline values over the whole dose range used $(\mathrm{P}<0.001$, paired Student $t$-test), and did not differ significantly from those obtained for control rats ( $P>0.05$, one-way ANOVA). In both malnourished groups, the pressor effects elicited by PHE evoked the expected reflex bradycardia. The relationship between peak decreases in HR and peak increases in MAP is shown in Figure 3A. The baroreceptor sensitivity, as indicated by the slope for re-

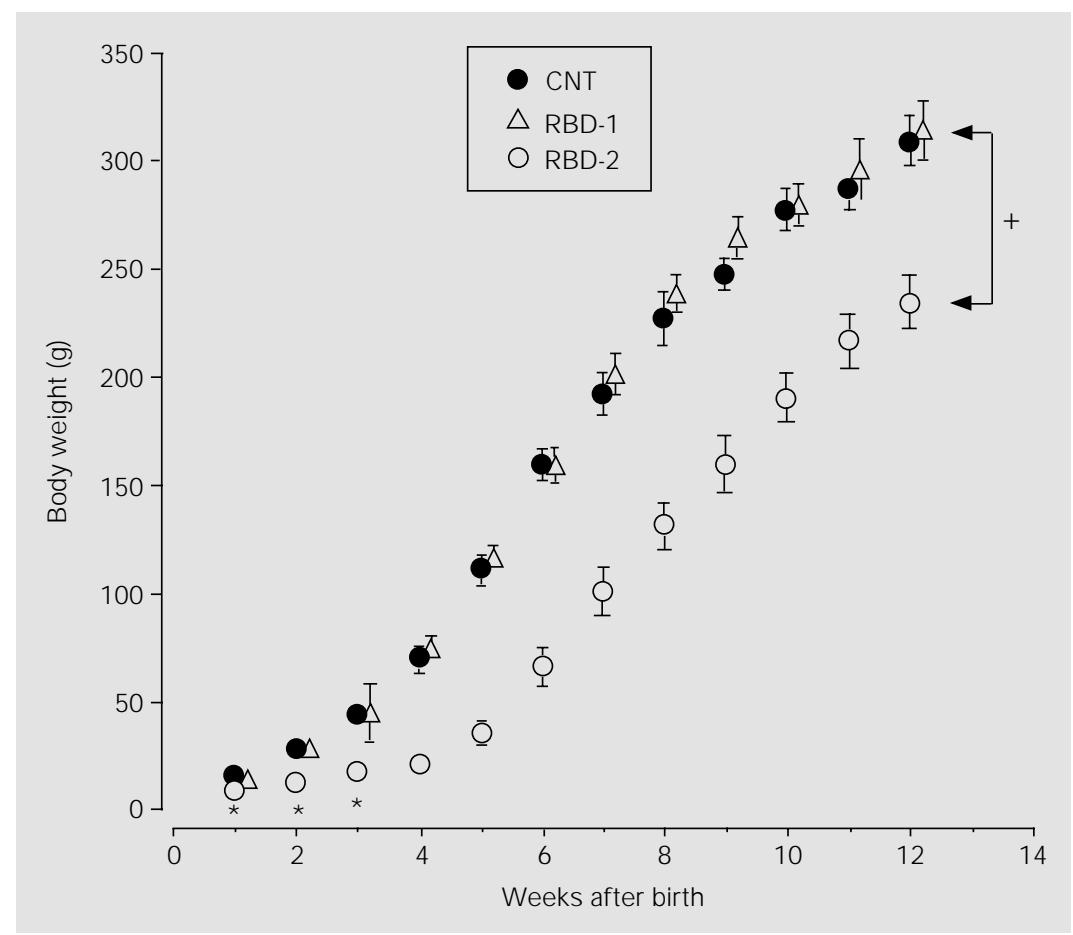

Figure 1 - Time course of body weight of offspring born to mothers receiving the regional basic diet (RBD) during pregnancy (RBD-1) or during nursing (RBD-2) and of those born to dams fed a standard balanced diet during pregnancy and nursing (CNT). Vertical bars indicate the SEM ( $\mathrm{N}=9$ rats per group). In all groups, body weight was determined weekly from the 1st to the 12th week of age. During this period, the mean body weight of RBD-1 rats was similar to that of CNT rats, while the mean body weight of RBD-2 rats was significantly ( ${ }^{+} \mathrm{P}<0.001$, two-way ANOVA) lower than that of CNT rats. ${ }^{*} \mathrm{P}<0.01$ compared to CNT (unpaired Student t-test). 
Figure 2 - Heart wet weight (HWW) (panel A), heart dry weight (HDW) (panel B) and HDW to body weight (BW) (panel C) ratio of 3-month-old rats born to mothers receiving the regional basic diet (RBD) during pregnancy (RBD-1) or during nursing (RBD-2), compared with those of age-matched rats born to dams fed a standard control diet during pregnancy and nursing (CNT). Vertical bars indicate the SEM ( $\mathrm{N}=9$ rats per group). Both wet and dry heart weights were significantly $(* P<0.05$, unpaired Student t-test) reduced in RBD-1 and RBD-2 rats. However, the HDW/BW ratio of both RBD- 1 and RBD-2 rats was comparable to that of CNT rats. flex bradycardia (bpm $/ \mathrm{mmHg}$ ), was comparable ( $\mathrm{P}>0.05$, Mann-Whitney U-test) for both RBD-1 (-2.08 \pm 0.11$)$ and RBD-2 (-2.32 $\pm 0.30)$ rats in relation to controls $(-2.09 \pm$ 0.10 ) (Figure 3B). In both groups of malnourished rats, SNP-induced depressor effects were associated with reflex tachycar-

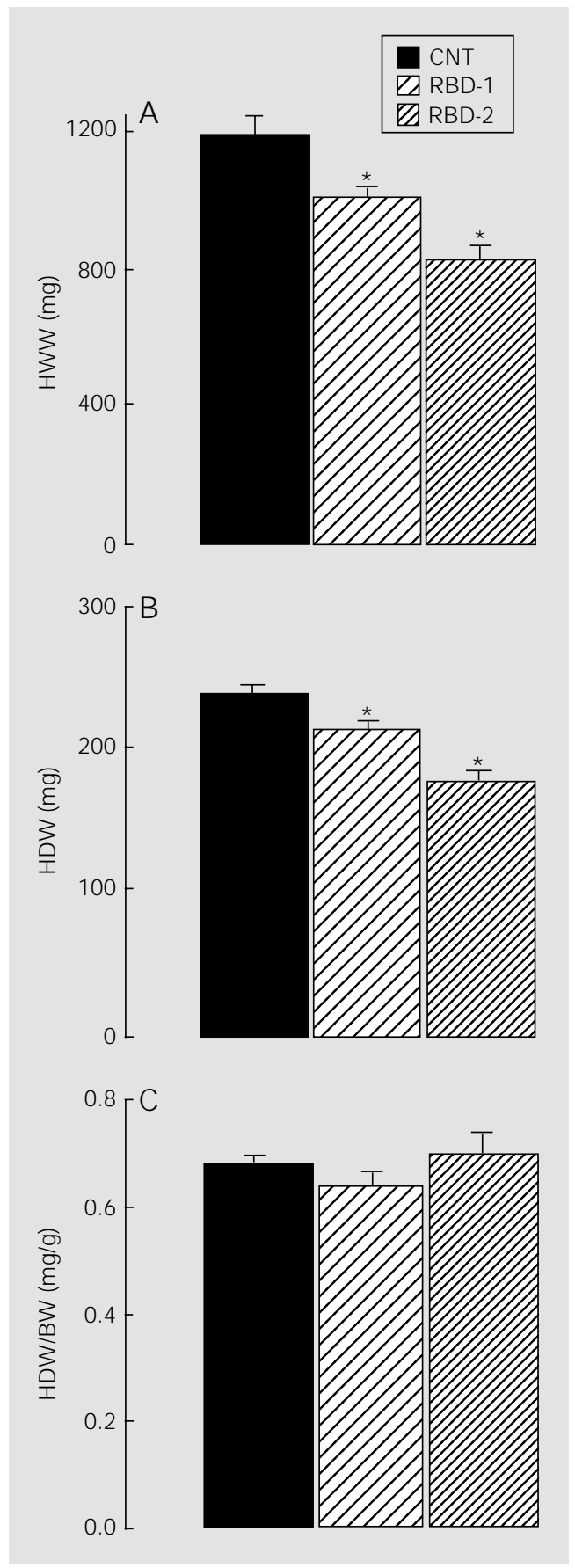

dia. The relationship between peak increases in HR and peak decreases in MAP is shown in Figure 4A. No significant differences ( $\mathrm{P}>0.05$, Mann-Whitney U-test) in reflex tachycardia responsiveness $(\mathrm{bpm} / \mathrm{mmHg}$ ) were detected between RBD-1 (-3.10 \pm 0.43$)$ or RBD-2 (-3.73 \pm 0.53$)$ and control $(-3.17 \pm$ 0.33 ) rats (Figure 4B), although reflex tachycardia tended to increase in RBD-2 rats (Figure $4 \mathrm{~A})$.

\section{Discussion}

The experimental diet used here is not only deficient in protein, but also in lipids, vitamins, sodium and other minerals (24, and present study). Given its higher carbohydrate content, the RBD is considered to be an isocaloric diet since its caloric adequacy was found to be comparable to that of the commercial control diet. Contrary to the findings of Teodósio et al. (24), in this study, daily food intake of mothers fed the RBD during pregnancy or nursing was lower than that of control mothers (F.M.F. Monteiro, S. Lahlou, J.A. Albuquerque and A.M.S. Cabral, unpublished results). Consequently, the daily protein and calorie intake of these malnourished mothers was lower than that of control mothers. Under such conditions, offspring of mothers fed the RBD during mating and pregnancy had lower birth weights, but displayed a weight gain similar to that of CNT rats from the 1st to the 12 th week of age. This finding contrasts with that of Langley and Jackson (19), who reported that offspring born to mothers receiving a low-protein diet (6\%) during pregnancy weighed significantly less over the whole monitoring period (from birth to 21 st week of age). This discrepancy may be related to differences in the feeding protocol since mothers in Langley's study were submitted to a low-protein diet not only during mating and pregnancy but also throughout the pre-mating period. Feeding mothers during the latter period would have markedly reduced maternal fat reserves, 
an effect which may have contributed to the lower body weight of pups in Langley's study. However, offspring of mothers fed the RBD during nursing had markedly lower body weights during the first week of age and were unable to catch up over the 12 week monitoring period. This finding is in agreement with a previous report (21), and supports the concept that postnatal nutritional manipulations affect adult size. Both wet and dry heart weights were significantly reduced in RBD-2 rats when compared to controls. This effect seems to parallel the reduction in body weight since no differences were detected when heart dry weight was related to body weight, a finding previously reported by other investigators $(31,32)$ using the restricted calorie model. However, both wet and dry heart weights were reduced in RBD-1 rats even when no significant reduction in body weight was detected in these animals at 12 weeks of age. This finding may suggest that when dams are fed the RBD during pregnancy, fetal heart development is affected in a negative and possibly irreversible fashion.

To the best of our knowledge, this is the first time that the effects of RBD-induced malnutrition on baseline blood pressure have been investigated. Our results show that although RBD-1 rats weighed significantly less at birth, their baseline MAP and HR recorded during adulthood were not significantly different from those of age-matched CNT rats. Similar results were also obtained for RBD-2 rats, although their body weight was lower throughout the 12-week monitoring period. Thus, these findings appear to disagree with the concept of an inverse relationship between birth weight and blood pressure in adult life. They may agree with those reported by Del Basso et al. (21), but these authors measured blood pressure in anesthetized rather than in conscious rats. The present results contrast with those of Langley and co-workers $(18,19)$ who showed that fetal exposure to maternal low-protein diets increased blood pressure in conscious rats, and that hypertension persisted into adulthood. Several methodological differences could help to explain this discrepancy. First, the composition of the diets varied not only with respect to the percentage of protein but also to the percentage of fats, carbohydrates, and salt. Thus, changes in dietary composition may have contributed to the result obtained here. For instance, given the well-known beneficial role of a low-sodium diet in essential hypertension (33), it is possible that the low sodium content of the RBD may account for the lack of increased blood pressure in the present study. Further experiments are underway in our laboratory to test this possibility, as well as the relative contribution of each deficient nutrient of the RBD. Second, the RBD is also quantitatively deficient in essential and non-essential amino acids (24). For instance, the essential amino

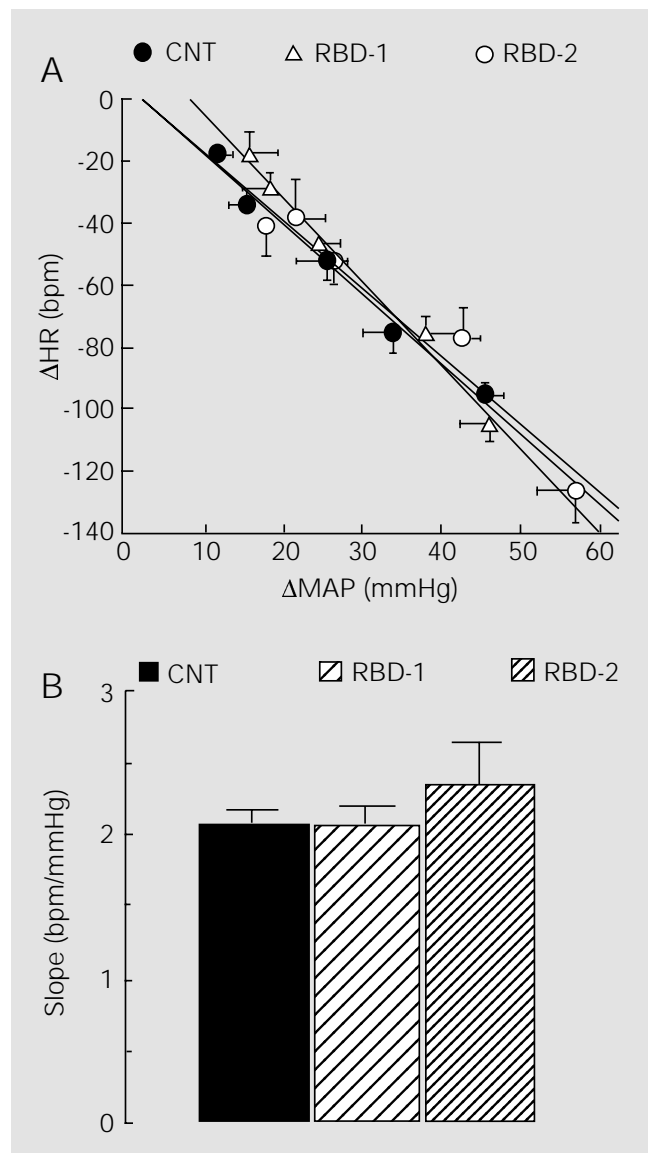

Figure 3 - A, Relationship between changes in mean arterial pressure $(\triangle \mathrm{MAP})$ and heart rate $(\triangle \mathrm{HR})$ following increasing bolus doses of phenylephrine in adult rats obtained by feeding dams a standard control diet during pregnancy and nursing (CNT) or by feeding them the regional basic diet (RBD) during pregnancy (RBD-1) or during nursing (RBD-2). B, Average slopes of the phenylephrine response in the above three groups. Vertical and horizontal bars indicate the SEM ( $\mathrm{N}=8-9$ rats per group). 
acid methionine was reported to be present at the lowest level (24). In Langley's study (19), the synthetic diet was supplemented with this amino acid. Third, the feeding protocol was also different since in Langley's study (19) the mothers were submitted to a low-protein diet not only during mating and pregnancy but also throughout the pre-mating period. Finally, Langley and co-workers $(18,19)$ used the stressful tail-cuff technique to record blood pressure whereas direct measurement of MAP was performed in the present study. It is well known that the indirect method is not without drawbacks. For instance, this method gives readings of only systolic blood pressure. Furthermore, restraint as a source of stress, degree and extent of prewarming may influence the final measurement of systolic arterial blood pressure. It is reasonable to suggest that stress may have played an important role in reveal-

Figure 4 - A, Relationship between changes in mean arterial pressure $(\triangle \mathrm{MAP})$ and heart rate $(\triangle \mathrm{HR})$ following increasing bolus doses of sodium nitroprusside in adult rats obtained by feeding dams a standard control diet during pregnancy and nursing (CNT) or by feeding them the regional basic diet (RBD) during pregnancy (RBD-1) or during nursing (RBD-2). B, Average slopes of the sodium nitroprusside response in the above three groups. Vertical and horizontal bars indicate the SEM $(\mathrm{N}=8-9$ rats per group).

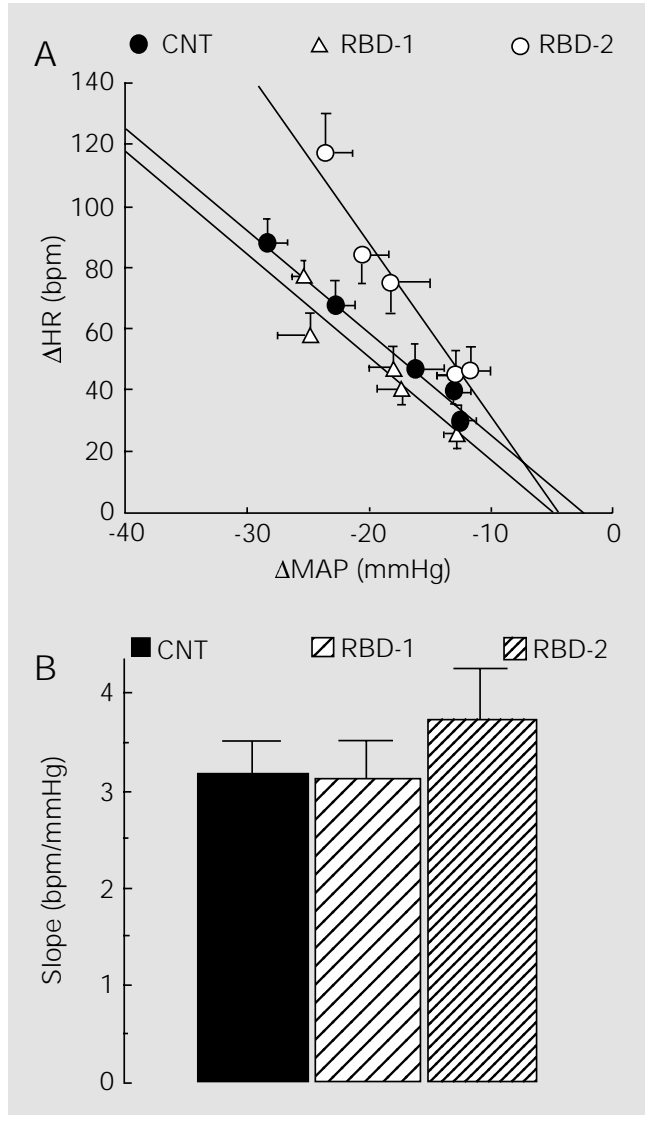

ing increased systolic blood pressure in prior investigations $(18,19)$. In this respect, using a direct and minimally stressful telemetric procedure, Tonkiss et al. (20) reported that while no statistical differences in systolic blood pressure were detected between prenatally malnourished and well-nourished control rats, malnourished rats showed a greater increase in both diastolic and systolic pressures compared with control rats during exposure to the olfactory stressor ammonia.

Although the effects of pre- or perinatal dietary manipulations on baroreflex responsiveness had not been tested before this study, the effects of long-term calorie restriction on this reflex have been studied. Herlihy and colleagues (22) reported that chronic calorie restriction in adulthood enhanced baroreflex responsiveness to SNP-induced hypotension, but not to PHE-induced pressor effects, in normotensive Fisher 344 rats. A similar enhancement of baroreflex sensitivity was also found in the calorie-restricted spontaneously hypertensive rat (23). In the present study, baroreflex responses to both PHE and SNP remained unaltered in RBD-1 rats. Similar results were observed in RBD-2 rats, although reflex tachycardia tended to increase in these animals. The lack of a significantly enhanced baroreflex response to SNP may be due to the wide variability of the individual slope in the RBD-2 group. Nevertheless, this tendency to increased reflex tachycardia appears to agree with the finding of Herlihy et al. (22), and may indicate that the nursing period is more sensitive to the effects of this type of malnutrition than the prenatal period.

In conclusion, the present study shows for the first time that, after a prolonged period of nutritional recovery, the feeding of the multideficient RBD during pregnancy or nursing had no effects on resting blood pressure or baroreflex sensitivity in adult rats. These findings do not support the concept of an inverse relationship between birth weight and blood pressure in adulthood. However, 
the decreased body weight and the tendency to increased reflex tachycardia in RBD-2 rats may suggest that this type of maternal malnutrition during lactation is more critical than during pregnancy. Measurement of arterial blood pressure in rats submitted to malnutrition over an extended period of time from an earlier age (4-6 weeks of age) would

be highly recommended.

\section{Acknowledgments}

Reviewing of the manuscript by Dr. S.D.

Aird is gratefully acknowledged.

\section{References}

1. Pissaia O, Rossi MA \& Oliveira J SM (1980). The heart in protein-calorie malnutrition in rats: morphological, electrophysiological and biochemical changes. J ournal of Nutrition, 110: 2035-2044.

2. Singh GR, Malathi KE, Kasliwal RR, Ommar A, Padmavati S \& Ramji S (1989). An evaluation of cardiac function in malnourished children by non-invasive methods. Indian Pediatrics, 26: 875-881.

3. Olubodun J OB (1992). Nutritional factors and heart failure in Nigerians with hypertensive heart disease. International J ournal of Cardiology, 35: 71-76.

4. Saraiva LR, Filho DB \& Nora AD (1992). O coração na criança portadora de desnutrição calórico-proteica. Arquivos Brasileiros de Cardiologia, 58: 353-357.

5. Wharton B (1991). Protein energy malnutrition: problems and priorities. Acta Paediatrica Scandinavica, 374: 5-14.

6. Lucas SRR, Silva VLC, Miraglia SM \& Gil FZ (1997). Functional and morphologic evaluation of offspring kidney after intrauterine undernutrition. Pediatric Nephrology, 11: 719-723.

7. Lucas A (1998). Programming by early nutrition: an experimental approach. J ournal of Nutrition, 128: 401S-406S.

8. Gennser G, Rymark P \& Isberg PE (1988). Low birth-weight and risk of high blood pressure in adulthood. British Medical J ournal, 296: 1498-1500.

9. Walker BR, McConnachie A, Noon JP, Webb DJ \& Watt GCM (1998). Contribution of parental blood pressures to association between low birth weight and adult high blood pressure: cross sectional study. British Medical J ournal, 316: 834837.

10. Barker DJ P, Osmond C, Golding J , Kuh D \& Wadsworth MEJ (1989). Growth in utero, blood pressure in childhood and adult life, and mortality from cardiovascular diseases. British Medical J ournal, 298: 564-567.
11. Barker DJ P, Bull AR, Osmond C \& Simmonds SJ (1990). Fetal and placental size and risk of hypertension in adult life. British Medical J ournal, 301: 259-262.

12. Law CM, Swiet $M$, Osmond $C$, Fayers PM, Barker DJ P, Cruddas AM \& Fall CHD (1993). Initiation of hypertension in utero and its amplification throughout life. British Medical J ournal, 306: 24-27.

13. Lurbe E, Redon J, Alvarez V, Durazo R, Gomez A, Tacons J \& Cooper RS (1996). Relationship between birth weight and awake blood pressure in children and adolescents in absence of intrauterine growth retardation. American J ournal of Hypertension, 9: 787-794.

14. Zureik M, Bonithon-Kopp C, Lecomte $E$, Siest $G \&$ Ducimetière $P$ (1996). Weights at birth and in early infancy, systolic pressure, and left ventricular structure in subjects aged 8 to 24 years. Hypertension, 27: 339-345.

15. Seidman DS, Laor A, Gale R, Stevenson DK, Mashiach S \& Danon YL (1991). Birth weight, current body weight, and blood pressure in late adolescence. British Medical J ournal, 302: 1235-1237.

16. Matthes JWA, Lewis PA, Davies DP \& Bethel J A (1994). Relation between birth weight at term and systolic blood pressure in adolescence. British Medical J ournal, 308: 1074-1077.

17. Lucas A \& Morley R (1994). Does early nutrition in infants born before term programme later blood pressure? British Medical J ournal, 309: 304-308.

18. Langley SC, Phillips GJ \& J ackson AA (1994). In utero exposure to maternal low protein diets induces hypertension in weanling rats, independently of maternal blood pressure changes. Clinical Nutrition, 13: 319-324.

19. Langley SC \& J ackson AA (1994). Increased systolic blood pressure in adult rats induced by fetal exposure to maternal low protein diets. Clinical Science, 86:
217-222.

20. Tonkiss J, Trzcinska M, Galler J R, RuizOpazo N \& Herrera VLM (1998). Prenatal malnutrition-induced changes in blood pressure. Dissociation of stress and nonstress responses using radiotelemetry. Hypertension, 32: 108-114.

21. Del Basso P, Keller EA, Salica C \& Orsingher OA (1983). Vascular reactivity in perinatally undernourished rats. European J ournal of Pharmacology, 87: 107111.

22. Herliny J T, Stacy C \& Bertrand HA (1992). Long-term calorie restriction enhances baroreflex responsiveness in Fisher 344 rats. American J ournal of Physiology, 263: H1021-H1025.

23. Pedrozo $H$, Bertrand HA \& Herlihy JT (1994). Caloric restriction alters arterial blood pressure and baroreflex responsiveness of the spontaneously hypertensive rat. Age, 17: 23-27.

24. Teodósio NR, Lago ES, Romani SAM \& Guedes RCA (1990). A regional basic diet from northeast Brazil as a dietary model of experimental malnutrition. Archivos Latinoamericanos de Nutrición, 4: 535547.

25. Teodósio NR, Lago ES, Medeiros MC, Ferreira LMP \& Santos ETV (1986). Carbohydrate metabolism in malnutrition induced by the regional basic diet. Fasting glycemia and liver glycogen in rats with RBD-induced malnutrition. Proceedings of the 1st International Symposium of Nutrition, Recife, PE, Brazil, October 7-10, 40-41.

26. da-Silva AT, Costa FBR, Costa JA, Teodósio NR, Cabral-Filho J E \& Guedes RCA (1987). Sciatic nerve conduction velocity of malnourished rats fed the human "basic regional diet" of the northeast of Brazil. Brazilian J ournal of Medical and Biological Research, 20: 383-392.

27. Guedes RCA, Andrade AFD \& Cabral-Filho J E (1987). Propagation of cortical spreading depression in malnourished rats: fa- 
cilitatory effect of dietary protein deficiency. Brazilian J ournal of Medical and Biological Research, 20: 639-642.

28. Knufman NM, van den Laarse A, Vliegen HW \& Brinkman CJ (1987). Quantification of myocardial necrosis and cardiac hypertrophy in isoproterenol-pretreated rats. Research Communications in Chemical Pathology and Pharmacology, 57: 15-32.

29. Lahlou S \& Pinto Duarte G (1998). Bromocriptine-induced tachycardia in conscious rats: blunted response following isoprote- renol pretreatment for 5 days. Acta Physiologica et Pharmacologica Therapeutica Latinoamericana, 48: 165-174.

30. Lahlou S, Lima GC, Leão-Filho CSC \& Pinto Duarte G (2000). Effects of longterm pretreatment with isoproterenol on bromocriptine-induced tachycardia in conscious rats. Canadian J ournal of Physiology and Pharmacology, 78: 260-265.

31. Shrader RE \& Zeman FJ (1969). Effect of matemal protein deprivation on morphological and enzymatic development of neonatal rat tissue. J ournal of Nutrition, 99: 401-412.

32. J ohnson J D \& Dunham T (1988). Protein turnover in tissues of the fetal rat after prolonged maternal malnutrition. Pediatric Research, 23: 534-538.

33. Cianciaruso $B$, Bellizzi $V$, Minutolo $R$, Tavera A, Capuano A, Conte $G \&$ De Nicola $L$ (1998). Salt intake and renal outcome in patients with progressive renal disease. Mineral and Electrolyte Metabolism, 24: 296-301. 